Binary trees, coproducts and integrable systems

This article has been downloaded from IOPscience. Please scroll down to see the full text article.

2010 J. Phys. A: Math. Theor. 43085215

(http://iopscience.iop.org/1751-8121/43/8/085215)

View the table of contents for this issue, or go to the journal homepage for more

Download details:

IP Address: 132.199.97.29

The article was downloaded on 25/05/2012 at 17:24

Please note that terms and conditions apply. 


\title{
Binary trees, coproducts and integrable systems
}

\author{
B Erbe ${ }^{1}$ and $\mathbf{H} \mathbf{J}$ Schmidt $^{2}$ \\ ${ }^{1}$ Department of Physics, University of Regensburg, Regensburg, Germany \\ ${ }^{2}$ Department of Physics, University of Osnabrueck, Osnabrueck, Germany \\ E-mail: bjoern.erbe@physik.uni-regensburg.de and hschmidt@uos.de
}

Received 31 August 2009, in final form 13 January 2010

Published 8 February 2010

Online at stacks.iop.org/JPhysA/43/085215

\begin{abstract}
We provide a unified framework for the treatment of special integrable systems which we propose to call 'generalized mean-field systems'. Thereby previous results on integrable classical and quantum systems are generalized. Following Ballesteros and Ragnisco, the framework consists of a unital algebra with brackets, a Casimir element and a coproduct which can be lifted to higher tensor products. The coupling scheme of the iterated tensor product is encoded in a binary tree. The theory is exemplified by the case of a spin octahedron. The relation to other generalizations of the coalgebra approach is discussed.
\end{abstract}

PACS numbers: $02.30 . \mathrm{Ik}, 75.10 . \mathrm{Jm}, 20.00,75.10 . \mathrm{Hk}$

\section{Introduction}

In classical mechanics 'complete integrability' can be precisely defined in terms of the Arnol'dLiouville theorem [1]. The corresponding generalization of this concept to quantum theory has not yet been achieved. Nevertheless, there exists a rich literature on integrable quantum systems under various headlines such as Yang-Baxter equations [2], algebraic Bethe ansatz [3] and quantum groups [4]. Aside from this mainstream of research there are different theories of integrable systems which could be characterized as 'generalized mean-field systems' (GMFS) $[5,6]$. The aim of this paper is to provide a general framework for the description of such systems.

The prototype of the systems in question is a spin system where the spins are (Heisenberg-) coupled to each other with equal strength. It turns out that each spin will move exactly as if it were under the influence of a uniform magnetic field. This justifies the above characterization as (generalized) 'mean-field systems'. The first generalizing step would be to consider systems which consist of uniformly coupled integrable subsystems. This property can be recursively applied. The underlying sequence of partial uniform couplings is most conveniently encoded in a binary tree, the leaves of which correspond to the smallest constituents of the system, see 


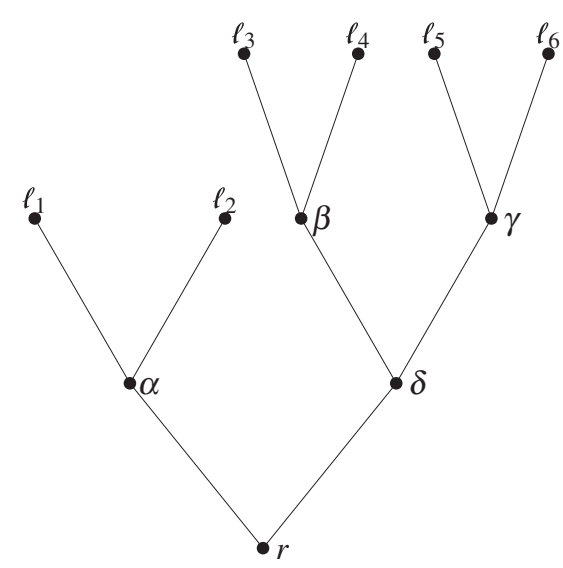

Figure 1. Example of a binary tree with root $r$, six leaves $\ell_{1}, \ldots, \ell_{6}$ and further nodes $\alpha, \beta, \gamma, \delta$.

[6]. For example, the uniform coupling of three pairs of spins can be described by the tree of figure 1 and gives rise to an integrable spin octahedron, see figure 5.

Another generalization into a different direction is based upon the insight that at the core a GMFS consists of a unital algebra $\mathcal{A}$ with a bracket [, \} and a co-multiplication $\Delta$, which can be lifted to tensor products of $\mathcal{A}$ and applied to a Casimir (central element) $c \in \mathcal{A}$, see [5]. One then considers representations of $\mathcal{A}$ generated by certain commutation relations where the bracket [, \} will either be represented by a Poisson bracket (classical case) or by the commutator of matrices (quantum case).

In our paper we simplify and generalize the approaches of $[5,6]$. Thereby the restriction to Heisenberg spin systems in [6] is abolished by incorporating the coalgebra ansatz of [5]. Vice versa, the theory of [5] will be reformulated by using the language of binary trees, and generalized from 'homogeneous trees' to general ones. We also found that the postulate in [5] of $\Delta$ being 'co-associative' is superfluous, but see appendix A. After the first publication of the coalgebra approach [5] various generalizations have been proposed, see [7-11]. We will comment on the relation of our approach to these generalizations at appropriate places in the paper and in two appendices. The obvious generalization of assuming several Casimir elements instead of a single one will be neglected here.

The paper is organized as follows. In section 2 we collect some definitions concerning binary trees which are needed later. Section 3 is devoted to the algebraic prerequisites including the coproduct $\Delta$ and its lift $\Delta^{T}$ to higher tensor products given by a binary tree $T$. In section 4 we apply these tools to the theory of integrable systems and prove the main result, theorem 1 , which is analogous to prop. 1 of [5] and provides a number of commuting observables which is in many examples sufficient to guarantee complete integrability. In section 5 we discuss the elementary example of a Heisenberg spin octahedron in order to illustrate the application of the abstract theory. Some remarks on the corresponding Gaudin spin system and on the connection to other approaches follow. Two appendices on the issues of superintegrability and the recent loop coproduct approach close the paper.

\section{Trees}

We consider finite, binary trees $T$, in short called 'trees'. Recall that these consist of a set of 'nodes' $\mathcal{N}(T)$, such that all nodes $n \in \mathcal{N}(T)$, except the 'leaves' $\ell \in \mathcal{L}(T)$, are connected to exactly two 'children' $c_{1}(n), c_{2}(n)$, mixing the metaphors of horticulture and genealogy. We 


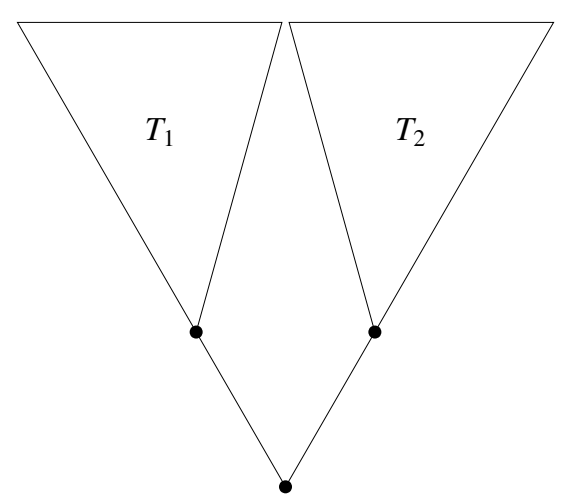

Figure 2. Union $\mathrm{V}\left(T_{1}, T_{2}\right)$ of two binary trees $T_{1}$ and $T_{2}$.

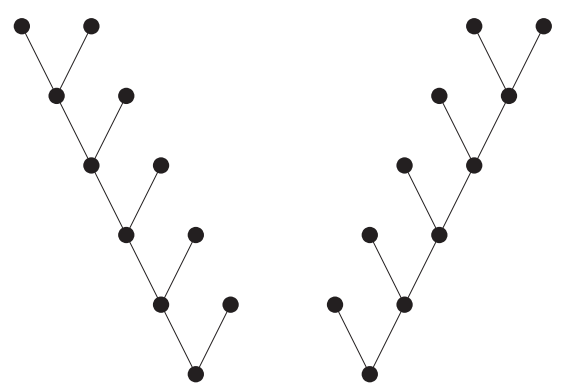

Figure 3. Examples of 'homogeneous' binary trees.

have to distinguish between the 'left child' $c_{1}$ and the 'right child' $c_{2}$. Due to this distinction, the leaves of a binary tree can be arranged in a natural order from left to right and hence be labeled by ' $\ell_{1}$ ' to ' $\ell_{L}$ '. All nodes, except the 'root' $r(T)$, are children of other nodes. By definition, different nodes have different children, see figure 1 . As a tree, $T$ is a connected graph without cycles.

The simplest tree $\bullet$ consists of only one root. The next simplest one $\mathrm{V}$ has three nodes, that is, one root and two leaves. If $T_{1}$ and $T_{2}$ are (disjoint) trees, then $\mathrm{V}\left(T_{1}, T_{2}\right)$ will be the tree obtained by identifying the leaves of $\mathrm{V}$ with the roots $r\left(T_{1}\right)$ and $r\left(T_{2}\right)$, see figure 2 .

Obviously, each tree can be obtained from copies of $\bullet$ by recursively applying the operation $\mathrm{V}\left(T_{1}, T_{2}\right)$. This opens the possibility of providing recursive definitions and proofs in the theory of trees. A tree $T$ will be called 'homogeneous' if it is of the form

$$
\begin{aligned}
& T=\mathrm{V}(\ldots(\mathrm{V}(\mathrm{V}(\bullet, \bullet), \bullet), \ldots, \bullet) \\
& \text { or } \quad T=\mathrm{V}(\bullet, \mathrm{V}(\bullet, \ldots, V(\bullet, \bullet) \ldots),
\end{aligned}
$$

see, for example, figure 3 . The tree of figure 1 is not homogeneous.

Binary trees are used in various parts of physics, e.g. in the chaos theory [12], computational physics [13] or in the theory of spin networks [14]. Here we utilize these structures for encoding the coupling schemes of certain integrable spin systems, similarly as in $[6,15,16]$. 


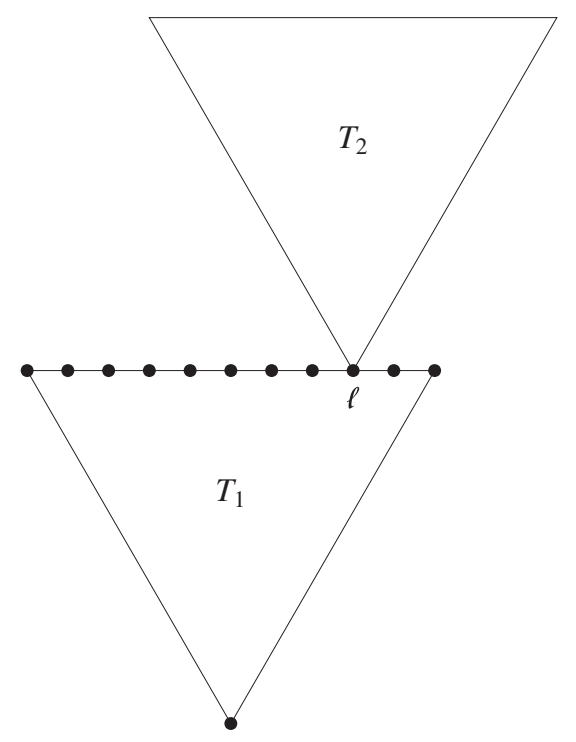

Figure 4. Binary tree $T=T_{1} \rho_{\ell} T_{2}$ obtained by grafting $T_{2}$ on $T_{1}$.

The following lemma can be easily proved.

Lemma 1. $N(T) \equiv|\mathcal{N}(T)|=2|\mathcal{L}(T)|-1 \equiv 2 L(T)-1$.

A sub-tree $S \subset T$ is given by a subset of nodes of $T$, which, according to their connections inherited from $T$, again form a tree. For example, if $n \in \mathcal{N}(T)$, then $T(n)$ will denote the maximal sub-tree of $T$ with the root $n$. Let $\mathcal{L}(n) \equiv \mathcal{L}(T(n))$. If $n, m \in \mathcal{N}(T)$, then either $\mathcal{L}(n) \subset \mathcal{L}(m)$ or $\mathcal{L}(m) \subset \mathcal{L}(n)$ or $\mathcal{L}(n) \cap \mathcal{L}(m)=\emptyset$. In the former two cases $m$ and $n$ will be called 'connected', in the latter case 'disjoint'.

If $T_{1}$ and $T_{2}$ are (disjoint) trees and $\ell \in \mathcal{L}\left(T_{1}\right)$, then $T=T_{1} \circ_{\ell} T_{2}$ will denote the tree obtained by 'grafting', i.e. by identifying the root $r\left(T_{2}\right)$ with the leaf $\ell$ of $T_{1}$, see figure 4.

\section{Coproducts}

In this paper we will often consider the classical and the quantum case simultaneously. In both cases, the physical observables are obtained by suitable representations of an abstract unital algebra $(\mathcal{A}, e)$ and its tensor products. In the quantum case, $\mathcal{A}$ will be an associative, nonabelian algebra with commutator $[a, b]=a b-b a, a, b \in \mathcal{A}$, and its physical representation will be given in terms of finite-dimensional matrices. Typical examples are cases where $\mathcal{A}$ is defined as the universal enveloping algebra of some semi-simple Lie algebra. In the classical case, $\mathcal{A}$ will be an Abelian algebra together with an abstract Poisson bracket $\{$,$\} , see [5].$ Representations of $\mathcal{A}$ are then given by the algebra of smooth functions of some phase space together with the usual Poisson bracket. To cover both cases, the commutator/Poisson bracket will be denoted by $[a, b\}, a, b \in \mathcal{A}$. It makes $(\mathcal{A},[\}$,$) into a Lie algebra and will act as an$ derivation on the associative product on $\mathcal{A}$. We will always consider algebras endowed with a bracket of one of these two kinds and the corresponding homomorphisms, that is, linear algebra homomorphism w.r.t. both multiplications. 
If $\mathcal{A}_{1}, \mathcal{A}_{2}$ are two algebras as explained above, then $\mathcal{A}_{1} \otimes \mathcal{A}_{2}$ will denote the algebraic tensor product, physically describing a composite system. It will be again a unital algebra with brackets upon linearly extending the definitions

$$
(a \otimes b)(c \otimes d)=(a c) \otimes(b d)
$$

and

$$
[(a \otimes b),(c \otimes d)\}=[a, c] \otimes \frac{b d+d b}{2}+\frac{a c+c a}{2} \otimes[b, d\} .
$$

If $A: \mathcal{A}_{1} \longrightarrow \mathcal{A}_{1}$ and $B: \mathcal{A}_{2} \longrightarrow \mathcal{A}_{2}$ are morphisms as explained above, then also $A \otimes B: \mathcal{A}_{1} \otimes \mathcal{A}_{2} \longrightarrow \mathcal{A}_{1} \otimes \mathcal{A}_{2}$ will be such a morphism. The 'coproduct' $\Delta$ will be a morphism

$$
\Delta: \mathcal{A} \longrightarrow \mathcal{A} \otimes \mathcal{A}
$$

that is, a linear algebra morphism plus a Poisson bracket morphism in the classical case. Usually, a coproduct is additionally required to be 'co-associative', see [5], but this property will not be needed in the main part of the present paper; hence, here we use the term 'coproduct' in a more general sense. In appendix A, co-associativity and co-commutativity of $\Delta$ will be assumed to extend the set of integrals of motion (superintegrability).

The crucial construction for integrability, as considered here, is the lift of the coproduct to higher order tensor products given by a tree $T$. To this end we first define $\mathcal{A}^{T}$ recursively by

$$
\begin{aligned}
& \mathcal{A}^{\bullet}=\mathcal{A} \\
& \mathcal{A}^{\mathrm{V}\left(T_{1}, T_{2}\right)}=\mathcal{A}^{T_{1}} \otimes \mathcal{A}^{T_{2}} .
\end{aligned}
$$

Sometimes it will be convenient to use the identification

$$
\mathcal{A}^{T_{1}}=\mathcal{A}^{T_{2}}=\underbrace{\mathcal{A} \otimes \cdots \otimes \mathcal{A}}_{L \text { times }},
$$

if $L\left(T_{1}\right)=L\left(T_{2}\right) \equiv L$. With respect to this identification the canonical embedding

$$
j_{n}: \mathcal{A}^{T(n)} \longrightarrow \mathcal{A}^{T}, \quad n \in \mathcal{N}(T)
$$

can be defined by

$$
j_{n}(a)=e \otimes \cdots \otimes a \otimes \cdots \otimes e, \quad a \in \mathcal{A}^{T(n)} .
$$

In the next step we define the lift of the coproduct $\Delta^{T}: \mathcal{A} \longrightarrow \mathcal{A}^{T}$ recursively by

$$
\begin{aligned}
& \Delta^{\bullet}=\operatorname{id}_{\mathcal{A}} \\
& \Delta^{\mathrm{V}\left(T_{1}, T_{2}\right)}=\left(\Delta^{T_{1}} \otimes \Delta^{T_{2}}\right) \circ \Delta,
\end{aligned}
$$

and conclude the following.

Lemma 2. $\Delta^{T}: \mathcal{A} \longrightarrow \mathcal{A}^{T}$ is a (Poisson) algebra morphism.

Proof. By induction over $T$. The claim follows since the tensor product and the composition of (Poisson) algebra morphisms is again a (Poisson) algebra morphism.

Before formulating the main result we still need another definition. Let $n \in \mathcal{N}(T)$, then

$$
\Delta^{n} \equiv j_{n} \circ \Delta^{T(n)}: \mathcal{A} \longrightarrow \mathcal{A}^{T} .
$$

We note that the generalization of the coalgebra approach to comodule algebras [7] where $\Delta^{n}$ is replaced by a suitable map $\mathcal{A} \longrightarrow \mathcal{A} \otimes \mathcal{B} \otimes \cdots \otimes \mathcal{B}$ is only possible for homogeneous trees. 


\section{Integrable systems}

Remember the grafting of trees $T=T_{1} \circ_{\ell} T_{2}$ explained in section 2. It gives rise to a corresponding composition of lifted coproducts in the following sense.

Lemma 3. Let $T=T_{1} \circ_{\ell} T_{2}, x \in \mathcal{A}$ and write $\Delta^{T_{1}}(x)=\sum_{i} x_{i, \ell_{1}} \otimes \cdots \otimes x_{i, \ell_{\mu}} \otimes \cdots \otimes x_{i, \ell_{L_{1}}}$, where $\ell_{1}, \ldots, \ell_{L_{1}}$ denote the leaves of $T_{1}$ and $\ell_{\mu}=\ell$. Then $\Delta^{T}(x)=\sum_{i} x_{i, \ell_{1}} \otimes \cdots \otimes$ $\Delta^{T_{2}}\left(x_{i, \ell_{\mu}}\right) \otimes \cdots \otimes x_{i, \ell_{L_{1}}}$.

Proof. By induction over $T_{1}$.

Further one can easily prove the following lemma.

Lemma 4. Let $n, m \in \mathcal{N}(T)$ be disjoint, i.e. $\mathcal{L}(n) \cap \mathcal{L}(m)=\emptyset$ and $x, y \in \mathcal{A}$. Then $\left[\Delta^{n}(x), \Delta^{m}(y)\right\}=0$.

Proof. $\Delta^{n}(x)$ and $\Delta^{m}(y)$ live in disjoint factors of the tensor product $\mathcal{A}^{T}$, since they are of the form $\Delta^{n}(x)=\sum_{i} e \otimes \cdots \bigotimes_{\ell \in \mathcal{L}(n)} x_{i, \ell} \otimes \cdots \otimes e$ and $\Delta^{m}(y)=\sum_{j} e \otimes \cdots \bigotimes_{\ell \in \mathcal{L}(m)} y_{j, \ell} \otimes \cdots \otimes e$. Hence, they commute.

Now we are ready to formulate the main result.

Theorem 1. Let $n, m \in \mathcal{N}(T)$ such that $\mathcal{L}(m) \subset \mathcal{L}(n), x \in \mathcal{A}$ and $c \in \mathcal{A}$ be a central element, i.e. $[y, c]=0$ for all $y \in \mathcal{A}$. Then $\left[\Delta^{n}(x), \Delta^{m}(c)\right\}=0$.

Proof. We introduce the canonical partial embedding $j_{m}^{n}: \mathcal{A}^{T(m)} \longrightarrow \mathcal{A}^{T(n)}$ such that $j_{m}=j_{n} \circ j_{m}^{n}$. It follows that

$$
\begin{aligned}
{\left[\Delta^{n}(x), \Delta^{m}(c)\right\} } & =\left[j_{n} \circ \Delta^{T(n)}(x), j_{m} \circ \Delta^{T(m)}(c)\right\} \\
& =j_{n}\left(\left[\Delta^{T(n)}(x), j_{m}^{n} \circ \Delta^{T(m)}(c)\right\}\right),
\end{aligned}
$$

and thus it suffices to show that

$$
\left[\Delta^{T(n)}(x), j_{m}^{n} \circ \Delta^{T(m)}(c)\right\}=0 .
$$

By applying lemma 3 to the sub-tree $T(n)$ we write

$$
T(n)=T_{1} \circ_{m} T(m), \quad m=\ell_{\mu} \in \mathcal{L}\left(T_{1}\right),
$$

and conclude

$$
\Delta^{T(n)}(x)=\sum_{i} x_{i, 1} \otimes \cdots \Delta^{T(m)}\left(x_{i, \mu}\right) \otimes \cdots x_{i, L_{1}} .
$$

Hence,

$$
\begin{aligned}
{\left[\Delta^{T(n)}(x), j_{m}^{n} \circ \Delta^{T(m)}(c)\right\} } & =\sum_{i} x_{i, 1} \otimes \cdots\left[\Delta^{T(m)}\left(x_{i, \mu}\right), \Delta^{T(m)}(c)\right\} \otimes \cdots x_{i, L_{1}} \\
& =\sum_{i} x_{i, 1} \otimes \cdots \Delta^{T(m)}\left(\left[x_{i, \mu}, c\right\}\right) \otimes \cdots x_{i, L_{1}} \\
& =0,
\end{aligned}
$$

since $\left[x_{i, \mu}, c\right\}=0$.

This theorem generalizes prop. 1 of [5] to arbitrary, not necessarily homogeneous trees. In order to guarantee complete integrability in the sense of the Arnol'd-Liouville theorem for $2 L$-dimensional phase spaces (which is satisfied for spin systems, see section 5 ) 
we would need $L$ pairwise commuting observables ('integrals in involution'). These are provided by the $\Delta^{n}(c)$ for each node $n$ which is not a leaf since theorem 1 and lemma 4 immediately imply $\left[\Delta^{n}(c), \Delta^{m}(c)\right\}=0$ for all $n, m \in \mathcal{L}(T)$. By lemma 1 , there are exactly $N(T)-L(T)=L(T)-1$ such nodes. In [5], the remaining observable is chosen as the Hamiltonian $H$. In the context of quantum spin systems another choice would be more appropriate, namely $\Delta^{r}(x)$ with a suitable $x \in \mathcal{A}$. The Hamiltonian $H$ could then be chosen as any element of the algebra generated by the $\Delta^{n}(c)$ and $\Delta^{r}(x)$, see section 5 . In the general case the dimension of the phase space depends on the symplectic realization of $(\mathcal{A},\{\}$,$) and$ the choice of the symplectic leaves. A thorough discussion of these questions, which also applies to the binary tree approach, including issues of superintegrability can be found in [9].

\section{Examples and outlook}

In order to explain the application of theorem 1 to the integrability of quantum systems we consider the elementary example of a spin octahedron, figure 5, with Heisenberg Hamiltonian, following [6]. We chose as $\mathcal{A}$ the universal enveloping algebra of the Lie algebra $S U(2)$. More concretely, we consider three generators $X_{1}, X_{2}, X_{3}$ satisfying the abstract commutations relations

$$
\left[X_{j}, X_{k}\right]=\mathrm{i} \sum_{\ell=1}^{3} \epsilon_{j k \ell} X_{\ell},
$$

where $\epsilon_{j k \ell}$ denotes the completely anti-symmetric Lévi-Cività symbol. $\mathcal{A}$ is the set of all finite polynomials $X$ of the standard form

$$
X=\sum_{k l m} c_{k l m} X_{1}^{k} X_{2}^{l} X_{3}^{m}
$$

The product $X Y$ of two such polynomials is brought into the standard form (21) by successively applying the commutation relations (20). The unit element in $\mathcal{A}$ is $e=X_{1}^{0} X_{2}^{0} X_{3}^{0}$. It follows that $c \equiv X_{1}^{2}+X_{2}^{2}+X_{3}^{2}$ commutes with all $X \in \mathcal{A}$.

The coproduct $\Delta$ is defined on the generators by

$$
\Delta\left(X_{i}\right)=e \otimes X_{i}+X_{i} \otimes e,
$$

and then extended to general elements of the form (21) by employing the property of $\Delta$ being an algebra homomorphism. Thus, for example,

$$
\begin{aligned}
\Delta(c) & =\Delta\left(X_{1}^{2}+X_{2}^{2}+X_{3}^{2}\right) \\
& =\Delta\left(X_{1}\right)^{2}+\Delta\left(X_{2}\right)^{2}+\Delta\left(X_{3}\right)^{2} \\
& =\sum_{i=1}^{3}\left(e \otimes X_{i}+X_{i} \otimes e\right)^{2} \\
& =e \otimes c+c \otimes e+2 \sum_{i=1}^{3} X_{i} \otimes X_{i} .
\end{aligned}
$$

We further choose $T$ as the binary tree of figure 1 and obtain the corresponding various commutation relations of theorem 1 being valid in the sixfold tensor product $\bigotimes_{i=1}^{6} \mathcal{A}$ where one usually chooses $\Delta^{r}(x)=\Delta^{r}\left(X_{3}\right)$. Next we consider the well-known $(2 s+1)$-dimensional irreducible matrix representation of (20) and denote the representations of the generators $X_{i}$ by $\mathbf{S}_{i}$ 'spin operator components'. In the sixfold tensor product we denote the single spin 


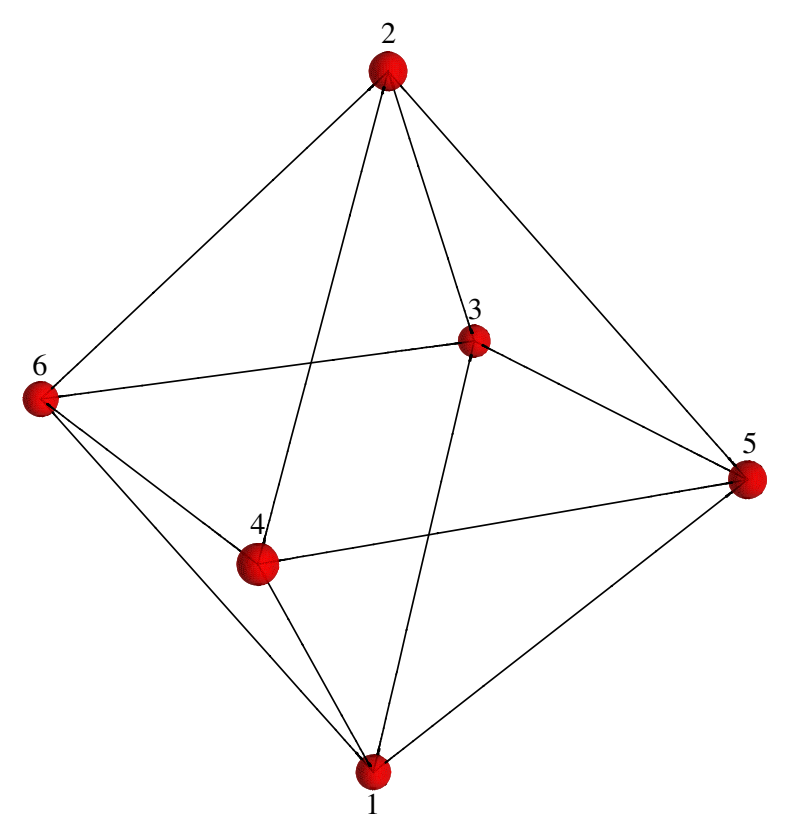

Figure 5. The octahedral spin graph corresponding to the integrable Heisenberg Hamiltonian (27). Its coupling scheme is encoded in the binary tree of figure 1 as explained in the text.

(This figure is in colour only in the electronic version)

components by $\mathbf{S}_{i}^{\mu}, \mu=1, \ldots, 6$. In this representation, $c=s(s+1) \mathbf{1}$ and all commutation relations of theorem 1 remain valid. Note that $\Delta(c)$ becomes $\left(\mathbf{S}^{(1)}+\mathbf{S}^{(2)}\right)^{2}$, which is no longer a constant, analogously for higher tensor products $\Delta^{n}(c)$. This shows, by the way, why it is advantageous to work in an abstract setting and to consider concrete representations only after the coproduct is defined. Furthermore, note that we could slightly generalize the example by considering different $s$ for each factor of the tensor product.

Let $(\mathcal{V}, \mathcal{E})$ be the the octahedral spin graph of figure 5 with its set of six vertices $\mathcal{V}$ and the set of 12 edges $\mathcal{E}$. The corresponding Heisenberg Hamiltonian $H$ can be written in various ways:

$$
\begin{aligned}
H & =2 J \sum_{(\mu, v) \in \mathcal{E}} \mathbf{S}^{(\mu)} \cdot \mathbf{S}^{(v)} \\
& =J\left(\left(\sum_{\mu \in \mathcal{V}} \mathbf{S}^{(\mu)}\right)^{2}-\left(\mathbf{S}^{(1)}+\mathbf{S}^{(2)}\right)^{2}-\left(\mathbf{S}^{(3)}+\mathbf{S}^{(4)}\right)^{2}-\left(\mathbf{S}^{(5)}+\mathbf{S}^{(6)}\right)^{2}\right) \\
& =J\left(\Delta^{r}(c)-\Delta^{\alpha}(c)-\Delta^{\beta}(c)-\Delta^{\gamma}(c)\right),
\end{aligned}
$$

where the root $r$ and the nodes $\alpha, \beta, \gamma$ refer to the binary tree of figure $1 . J$ is some appropriate coupling constant. It is crucial that $H$ can be written as a linear combination of commuting observables according to theorem 1. In this respect the octahedral Heisenberg Hamiltonian (27) is only the simplest case; for example, a Zeeman term proportional to $\Delta^{r}\left(\mathbf{S}_{3}\right)$ could be added without loosing integrability. The eigenvalues and common eigenvectors of the system of commuting observables $\Delta^{n}(c), n \in \mathcal{N}(T)$, result from the well-known rules of 
coupling angular momenta involving Clebsch-Gordan coefficients. An explicit formula for the eigenvalues and eigenvectors of $H$ and arbitrary binary trees has been given in [6]. The example of the spin octahedron clearly shows the physical meaning of the binary tree $T$ on which theorem 1 depends: $T$ encodes the coupling scheme of systems which are completely integrable due to their structure of uniformly coupled subsystems.

With exactly the same algebraic considerations and the same tree as above, theorem 1 provides us with another very interesting integrable model,

$$
\begin{aligned}
H & =A\left(\mathbf{S}^{(1)} \cdot \sum_{i=3}^{6} \mathbf{S}^{(i)}+\mathbf{S}^{(2)} \cdot \sum_{i=3}^{6} \mathbf{S}^{(i)}\right)+2(A+J) \mathbf{S}^{(1)} \cdot \mathbf{S}^{(2)} \\
& =A\left(\Delta^{r}(c)-\Delta^{\delta}(c)\right)+J \Delta^{\alpha}(c)
\end{aligned}
$$

The (Gaudin) Hamiltonian $H$ describes a central spin system with two central spins of exchange $2(A+J)$, coupled homogenously to a bath of four spins. Such a system can serve for example as a simplified model for the hyperfine interaction in a double quantum dot, see [17].

Apart from this physical meaning, it is interesting from a formal point of view. Besides the approach presented in this paper, systems can be integrable in the sense of algebraic Bethe ansatz. According to the ground breaking work of Drinfeld [4], this is based on quasicocommutative bialgebras, which essentially means that there is an element $R \in \mathcal{A} \otimes \mathcal{A}$ with

$$
(\tau \circ \Delta)(x) \cdot R=R \cdot \Delta(x)
$$

for all $x \in \mathcal{A}$. $\tau$ denotes the switch operator defined by linearly extending $\tau(a \otimes b)=b \otimes a$ and $\Delta$ a usual coproduct.

As this algebraic structure is somewhat similar to the one presented in this paper, the question arises whether there is a connection between the systems integrability in either sense. The above system, in contrast to the central spin system with one central spin, is not integrable by means of the algebraic Bethe ansatz. Hence, adding a second central spin destroys the Bethe ansatz, whereas the integrability in the sense of theorem 1 remains unaffected.

Recently, a framework for integrability using the so-called 'loop coproducts' has been proposed $[10,11]$ which contains different previous approaches to integrability as special cases. It is, however, confined to the classical case. Some remarks on the relation between this approach and the present paper are included in appendix B.

\section{Acknowledgments}

We are indebted to a referee for suggestions to the literature concerning generalizations of [5] and to Roman Schnalle for references on coupling trees.

\section{Appendix A. Superintegrability}

The approach [5] to integrability via coalgebras has subsequently been extended to 'superintegrability' [8,9]. This roughly means that one is seeking for additional integrals of motion which, however, do not longer commute with the old ones. 'Additional' means, in the classical case, that the new integrals of motion are functionally independent of the old ones. Typically, this functional independence cannot be shown in the general setting, but only in concrete examples, see $[8,9]$. Also in theses references the role of co-associativity of $\Delta$ in connection to superintegrability has been stressed.

The question arises whether these ideas can be transferred to the more general situation where the binary trees are not necessarily of homogeneous type. To this end we slightly refine 

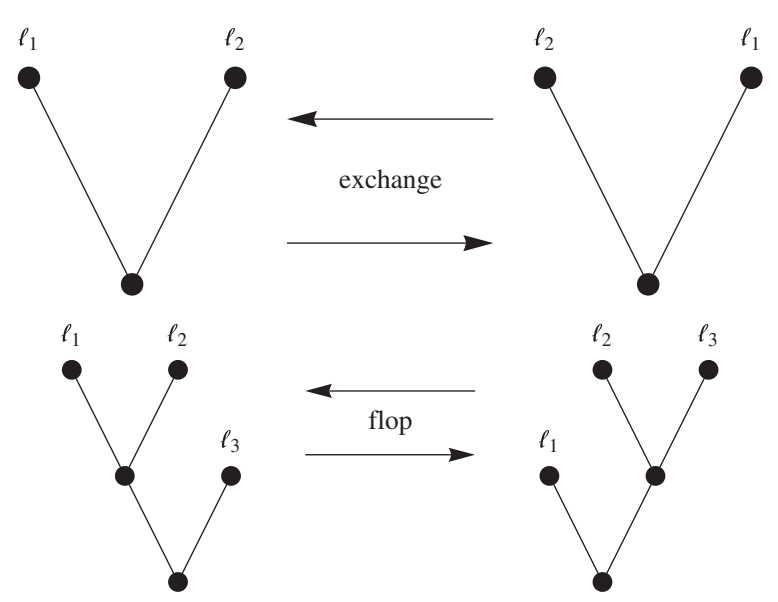

Figure A1. Illustration of the operations 'exchange' and 'flop' on coupling trees.

the binary tree construct, following, for example, [15]. Recall that, due to the distinction between 'left child' and 'right child', the leaves of a binary tree can be arranged in a natural order from left to right and hence be labeled by ' $\ell_{1}$ ' to ' $\ell_{L}$ '. Now assume that this labeling can be arbitrarily permuted. We will call the resulting structure a coupling tree. For example, the only binary tree with three nodes, $\mathrm{V}$, gives rise to two different coupling trees, denoted by $\mathrm{V}\left(\ell_{1}, \ell_{2}\right)$ and $\mathrm{V}\left(\ell_{2}, \ell_{1}\right)$. Generally, a coupling tree $\tilde{T}$ can be represented as a pair $\tilde{T}=(T, \pi)$, where $T$ is a binary tree with $L$ leaves and $\pi \in \mathcal{S}_{L}$, a permutation of $L$ elements. A coupling tree with $L$ leaves can alternatively be construed as a monomic expression in the abstract variables $\ell_{1}, \ldots, \ell_{L}$, such that each variable occurs exactly once. For example, the tree of figure 1 , conceived as a coupling tree, corresponds to the expression $\left(\ell_{1} \ell_{2}\right)\left(\left(\ell_{3} \ell_{4}\right)\left(\ell_{5} \ell_{6}\right)\right)$.

Most definitions and propositions of sections 2 and 3 can be taken over directly or with minor modifications. It will be appropriate to reserve the union and grafting operations, see figures 2 and 4, to binary trees, and to obtain the corresponding coupling trees by adducing a suitable permutation of the leaves, as explained above. Note that the definition of $\mathcal{A}^{T}$ remains unchanged. We will extend definitions (6) and (7) to coupling trees by

$$
\Delta^{\tilde{T}}=\tilde{\pi} \circ \Delta^{T}
$$

where $\tilde{T}=(T, \pi)$ and $\tilde{\pi}: \mathcal{A}^{T} \longrightarrow \mathcal{A}^{T}$ denotes the natural representation of $\pi$ by a permutation of factors of the tensor product. Following [15] we consider two operations on coupling trees, namely

$$
\begin{aligned}
& \text { exchange: } \mathrm{V}\left(\ell_{1}, \ell_{2}\right) \rightleftarrows \mathrm{V}\left(\ell_{2}, \ell_{1}\right) \\
& \text { flop: } \left.\mathrm{V}\left(\mathrm{V}\left(\ell_{1}, \ell_{2}\right), \ell_{3}\right) \rightleftarrows \mathrm{V}\left(\ell_{1}, \mathrm{~V}\left(\ell_{2}, \ell_{3}\right)\right)\right),
\end{aligned}
$$

see figure A1. We have the following.

Proposition 1. Let $T_{1}$ and $T_{2}$ be two coupling trees with $\mathcal{L}\left(T_{1}\right)=\mathcal{L}\left(T_{2}\right)$. Then $T_{1}$ can be transformed into $T_{2}$ by a finite sequence of exchanges and flops operating on subtrees.

We will skip the proof which is lengthy but straightforward. Note that, in the language of monomials, the proposition says that any two monomials with the variables $\ell_{1}, \ldots, \ell_{L}$ occurring exactly once can be transformed into each other by applying the rules of commutativity and associativity of the multiplication. 
We will say that the coproduct $\Delta: \mathcal{A} \longrightarrow \mathcal{A} \otimes \mathcal{A}$ is co-commutative iff $\Delta^{\mathrm{v}\left(\ell_{1}, \ell_{2}\right)}=$ $\Delta^{\mathrm{V}\left(\ell_{2}, \ell_{1}\right)}$ and co-associative iff $\Delta^{\mathrm{V}\left(\mathrm{V}\left(\ell_{1}, \ell_{2}\right), \ell_{3}\right)}=\Delta^{\mathrm{V}\left(\ell_{1}, \mathrm{~V}\left(\ell_{2}, \ell_{3}\right)\right)}$. Note that the coproduct defined in (22) is co-commutative as well as co-associative. Obviously, $\Delta$ is co-commutative and coassociative iff $\Delta^{T}$ is invariant under exchanges and flops operating on sub-trees of $T$. Together with proposition 1 we obtain the following.

Proposition 2. If $\Delta$ is co-commutative and co-associative and $\mathcal{L}\left(T_{1}\right)=\mathcal{L}\left(T_{2}\right)$ then $\Delta^{T_{1}}=\Delta^{T_{2}}$. Moreover, if $n_{1} \in \mathcal{N}\left(T_{1}\right)$ and $n_{2} \in \mathcal{N}\left(T_{2}\right)$ such that $\mathcal{L}\left(n_{1}\right)=\mathcal{L}\left(n_{2}\right)$, then $\Delta^{n_{1}}=\Delta^{n_{2}}$.

Now let $\mathcal{L}\left(T_{1}\right)=\mathcal{L}\left(T_{2}\right)$ and consider the involutive sub-algebra $\mathcal{C}_{1} \subset \mathcal{A}^{T_{1}}$ generated by the elements $\Delta^{n}(c), n \in \mathcal{N}\left(T_{1}\right)$ and $\Delta^{r_{1}}(x)$, analogously for $\mathcal{C}_{2} \subset \mathcal{A}^{T_{2}}$, see section 4 . Define

$$
C N\left(T_{1}, T_{2}\right)=\left\{\left(n_{1}, n_{2}\right) \mid n_{1} \in \mathcal{N}\left(T_{1}\right), n_{2} \in \mathcal{N}\left(T_{2}\right), \mathcal{L}\left(n_{1}\right)=\mathcal{L}\left(n_{2}\right)\right\}
$$

and $\mathcal{C}_{12}$ as the sub-algebra of $\mathcal{C}_{1} \cap \mathcal{C}_{2}$ generated by the elements $\Delta^{n_{1}}(c), \Delta^{r_{1}}(x)$ or, equivalently, by the $\Delta^{n_{2}}(c), \Delta^{r_{2}}(x)$, where $\left(n_{1}, n_{2}\right)$ runs through $C N\left(T_{1}, T_{2}\right)$. Then we conclude the main result of this appendix.

Theorem 2. Let $\Delta$ be co-commutative and co-associative and $H \in \mathcal{C}_{12}$; then $[H, K\}=0$ for all $K \in \mathcal{C}_{1} \cup \mathcal{C}_{2}$.

The scenario for superintegrability considered in [8, 9] results as a special case of theorem 2 in the following sense. Let $T_{1}$ be the 'left-homogeneous tree' and $T_{2}$ the 'righthomogeneous tree' represented in figure 3. Then $C N\left(T_{1}, T_{2}\right)=\left\{\left(r_{1}, r_{2}\right)\right\}$ and $\mathcal{C}_{12}$ is the algebra generated by $\Delta^{r_{1}}(x)=\Delta^{r_{2}}(x)$. Note that in this case $T_{1}$ can be transformed into $T_{2}$ using only flops operating on sub-trees; hence, the assumption of $\Delta$ being co-commutative will be superfluous.

\section{Appendix B. Loop coproducts}

Recently, a framework for integrability using the so-called loop coproducts has been proposed by Musso $[10,11]$ which contains different previous approaches to integrability as special cases, namely the coalgebra approach [5], the linear $r$-matrix formulation and formulations using Sklyanin or reflection algebras. It is, however, confined to the classical case. Nevertheless, one may ask whether, in the classical case, the loop coproduct approach also includes the generalization of the coalgebra approach we have given in this paper.

At first glance, the answer seems to be 'no', since the corresponding derivation in [10] of the coalgebra as a special case utilizes the co-associativity of $\Delta$, which is not needed in our theorem 1 . Here we neglect the differences due to the assumption in $[10,11]$ that the algebra $\mathcal{A}$ has a finite number of generators. A closer inspection, however, reveals that co-associativity is not necessary.

The loop coproduct approach [11] is based on a family of maps $\Delta^{(k)}: \mathcal{A} \longrightarrow \mathcal{B}, k=$ $1, \ldots, m$, and postulates different properties of these maps for the cases $i<k$ (or $k<i$ ) and $i=k$. For comparison we have to set $\mathcal{B}=\mathcal{A}^{T}$. In our approach the set of nodes is only partially ordered by the definition $i \prec k$ iff $\mathcal{L}(i) \subset \mathcal{L}(k)$. However, $\prec$ can be extended to a linear order $<$ such that $i<k$ implies $\mathcal{L}(i) \subset \mathcal{L}(k)$. For $i<k$ we have either $\mathcal{L}(i) \cap \mathcal{L}(k)=\emptyset$ or $\mathcal{L}(i) \subset \mathcal{L}(k)$. In the first case $\left[\Delta^{i}(x), \Delta^{k}(y)\right\}=0$ for all $x, y \in \mathcal{A}$ due to lemma 4 . In the second case lemma 3 implies

$$
\left[\Delta^{i}(x), \Delta^{k}(y)\right\}=\sum_{j} f_{j}\left[\Delta^{i}\left(x_{j}\right), \Delta^{i}(y)\right\}
$$


for all $x, y \in \mathcal{A}$ and some suitable $f_{j} \in \mathcal{B}, x_{j} \in \mathcal{A}$. Hence, in both cases condition (4) of [11] is satisfied. If $i=k$, condition (5) of [11] follows since $\Delta^{i}$ is a (Poisson) algebra homomorphism in our theory.

We conclude that, in the case of classical mechanics and up to minor differences in the formulations, the loop coproduct theory $[10,11]$ contains the binary tree approach as a special case. Nevertheless, the binary tree approach has, to our opinion, its virtues as a constructive method particularly adapted to quantum spin systems.

\section{References}

[1] Arnol'd V I 1978 Mathematical Methods of Classical Mechanics (Berlin: Springer)

[2] Baxter R 1982 Exactly Solved Models in Statistical Mechanics (New York: Academic)

[3] Faddeev L D 1995 How algebraic Bethe Ansatz works for integrable model Les Houches lectures (arXiv:hep-th/9605187)

[4] Drinfeld V G 1987 Quantum groups Proc. Int. Congress Math. (Berkeley, CA, 1986) ed A M Gleason pp 798-820

[5] Ballesteros A and Ragnisco O 1998 A systematic construction of completely integrable Hamiltonians from coalgebras J. Phys. A: Math. Gen. 31 3791-813

[6] Steinigeweg R and Schmidt H-J 2009 Heisenberg-integrable spin systems Math. Phys. Anal. Geom. 12 19-45

[7] Ballesteros A, Musso F and Ragnisco O 2008 Comodule algebras and integrable systems J. Phys. A: Math. Theor. 41304028

[8] Ballesteros A, Herranz F J, Musso F and Ragnisco O 2004 Superintegrable deformations of the SmorodinskyWinternitz Hamiltonian Superintegrability in Classical and Quantum Systems (CRM Proceedings \& Lecture Notes vol 37) ed P Tempesta et al (Providence, RI: American Mathematical Society)

[9] Ballesteros A and Blasco A $2008 \mathrm{~N}$-dimensional superintegrable systems from symplectic realizations of Lie coalgebras J. Phys. A: Math. Theor. 41304028

[10] Musso F 2009 Loop coproducts arXiv:nlinSI/0907.4927

[11] Musso F 2009 Generalization of the r-matrix formulation through loop coproducts arXiv:nlinSI/0910.1047

[12] Ketoja J A and Kurkijärvi J 2009 Binary tree approach to scaling in unimodal maps J. Stat. Phys. 75 643-68

[13] Waltz J, Page G L, Milder S D, Wallin J and Antuns A 2002 A performance comparison of tree data structures for $N$-body simulation J. Comput. Phys. 178 1-14

[14] Tufarelli T and Giovannetti V 2009 High fidelity state transfer in binary tree spin networks Phys. Rev. A 79022313

[15] Fack V, Pitre S N and Van der Jeugt J 1994 New efficient programs to calculate general recoupling coefficients. Part I: generation of a summation formula Comput. Phys. Commun. 83 275-92

[16] Schnalle R and Schnack J 2009 Numerically exact and approximate determination of energy eigenvalues for antiferromagnetic molecules using irreducible tensor operators and general point-group symmetries Phys. Rev. B 79104419

[17] Schliemann J, Khaetskii A and Loss D 2003 Electron spin dynamics in quantum dots and related nanostructures due to hyperfine interaction with nuclei J. Phys.: Condens. Matter 15 1809-33 